\title{
Surface Diffusion of CO on Ni(111) Studied by Diffraction of Optical Second-Harmonic Generation off a Monolayer Grating
}

\author{
X. D. Zhu, Th. Rasing, and Y. R. Shen \\ Department of Physics, University of California, Materials and Chemical Sciences Division, \\ Lawrence Berkeley Laboratory, Berkeley, California 94720
}

(Received 8 August 1988)

\begin{abstract}
Diffraction of optical second-harmonic generation from a monolayer grating created by laser desorption of adsorbates can be used to study surface diffusion of molecules on substrates. Application of this novel technique to $\mathrm{CO}$ on $\mathrm{Ni}(111)$ yields a diffusion activation energy of $6.9 \mathrm{kcal} / \mathrm{mol}$ and a preexponential factor of $=1.2 \times 10^{-5} \mathrm{~cm}^{2} \mathrm{~s}^{-1}$.

PACS numbers: $68.35 . \mathrm{Fx}$, 68.35.Bs, $82.65 . \mathrm{Dp}$, 82.65.Jv
\end{abstract}

Surface diffusion is a subject of great importance in modern surface science. ${ }^{1-3}$ It often plays a major role in limiting a surface reaction. ${ }^{3,4}$ While molecular adsorption and desorption probe the potential variation perpendicular to the surface, surface diffusion probes the potential variation in the surface. Adsorbate-adsorbate and adsorbate-substrate interactions are expected to have different effects on desorption and on surface diffusion. ${ }^{5}$ The classical and quantum natures of surface diffusion have also attracted much attention. ${ }^{6}$ Despite the tremendous interest in the process, research in this area is still rather limited because of the lack of convenient tools. ${ }^{1}$ Field-ion microscopy is only applicable to the study of diffusion of single atoms on metal tips. ${ }^{7} \mathrm{Nu}$ clear magnetic resonance, limited by its sensitivity, requires porous substrates with a high surface-to-volume ratio. ${ }^{8}$ Field emission ${ }^{9}$ and Auger-electron microscopy ${ }^{10}$ have the difficulty of possibly perturbing the diffusion process. Work function measurements ${ }^{11}$ need special sample preparation for reliable analysis. Clearly, new and more versatile tools for surface diffusion studies are in great demand.

Recently, laser-induced thermal desorption has been developed as a very attractive technique to probe surface diffusion. ${ }^{12}$ A strong laser pulse is used to burn a hole in the adsorbed molecular monolayer. Subsequent timedelayed laser pulses are employed to desorb molecules that have diffused into the hole. The number of desorbed molecules by an individual laser pulse is recorded by a calibrated mass spectrometer. This technique, however, has the disadvantage of being quite complex in the data analysis, subject to uncertainty in the desorption profile and possible accumulative laser-induced surface damage. Furthermore, for a practical hole dimension $(-100 \mu \mathrm{m})$, it only allows the monitoring of relative fast diffusion processes (those which have a diffusion energy several times smaller than the desorption energy so that significant diffusion can happen without noticeable desorption before the surface becomes contaminated). The technique also does not yield any information about anisotropy of the diffusion process on a crystalline sur- face. ${ }^{13}$ In this Letter, we introduce a laser-induced surface grating technique to surmount these difficulties.

In our technique, two pulsed laser beams intersecting on the surface under investigation are used to burn a grating by thermal desorption of an adsorbed molecular monolayer. Subsequently, diffraction of surface optical second-harmonic generation (SHG) ${ }^{14}$ from the grating is used to detect the smearing of the grating due to surface diffusion of the adsorbed molecules. We have applied the technique to $\mathrm{CO}$ on $\mathrm{Ni}(111)$ in ultrahigh vacuum. The preliminary results allow us to deduce a diffusion energy $E_{\text {diff }}=6.9 \mathrm{kcal} / \mathrm{mol}$ with a preexponential factor $D_{0}=1.2 \times 10^{-5} \mathrm{~cm}^{2} / \mathrm{s}$.

Our experiment was performed in an ultrahigh vacuum chamber with a base pressure of $0.9 \times 10^{-10}$ Torr. A $\mathrm{Ni}(111)$ sample, $1 \mathrm{~cm}$ in diameter and $1.5 \mathrm{~mm}$ in thickness, was vertically mounted with one of its (110) axes oriented at $30^{\circ}$ off the horizontal plane. The sample was routinely cleaned by $\mathrm{Ar}^{+}$sputtering, followed by annealing during slow cooling down to room temperature. The surface showed little traces of $\mathrm{C}$ and $\mathrm{O}$ and less than $0.5 \%$ of a monolayer of $S$ as examined with Auger spectroscopy. Sharp $(1 \times 1)$ LEED patterns of $\mathrm{Ni}(111)$ indicated that the surface was well ordered. A ChromelAlumel thermal couple welded to the sample was used to monitor the sample temperature. For the diffusion experiment, the $\mathrm{Ni}(111)$ surface was initially dosed with CO to a coverage $\theta \sim \theta_{s} \sim 0.5$. The experiment was conducted at temperatures between 219 and $273 \mathrm{~K}$.

The optical arrangement (Fig. 1) involved a singlemode $Q$-switched $\mathrm{Nd}$-doped yttrium aluminum garnet laser with a FWHM pulsewidth of $16 \mathrm{~ns}$ at $1.06 \mu \mathrm{m}$. For the creation of a monolayer grating by laser desorption, the $1.064-\mu \mathrm{m}$ beam was first split into two and then recombined at incident angles $\phi= \pm 1.50^{\circ}$ to overlap on the $\mathrm{CO}$ covered $\mathrm{Ni}(111)$ surface over an area of $2-3 \mathrm{~mm}$ in diameter. Desorption of $\mathrm{CO}$ by the spatially modulated beam intensity as a result of interference produced a monolayer grating with a spacing $2 a=\lambda / 2 \sin \phi \sim 20 \mu \mathrm{m}$. Care was taken to ensure the absence of laser-induced surface damage. Details of the preparation of this mono- 


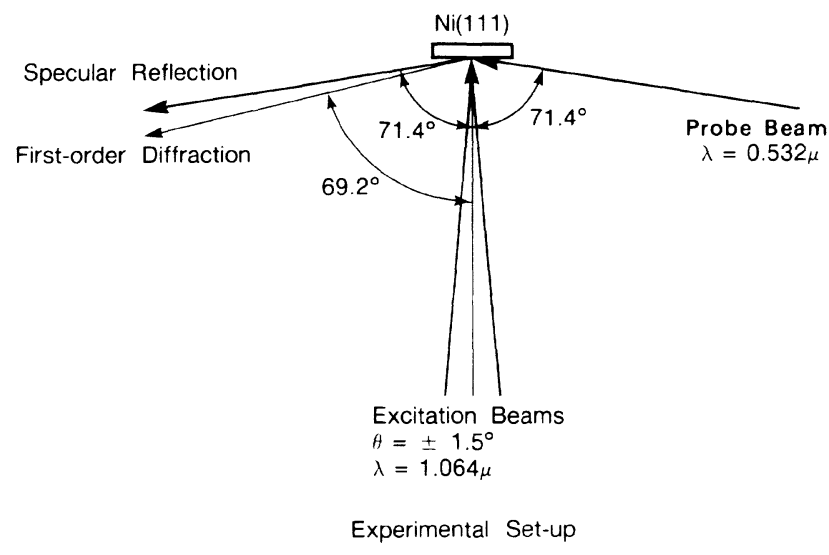

FIG. 1. The experimental setup for the surface diffusion measurement. The Ni(111) sample was placed in an ultrahigh vacuum chamber. The two excitation beam overlap and set up an interference pattern on the sample surface covered by $\mathrm{CO}$, generating a $\mathrm{CO}$ monolayer grating via thermal desorption. The probe beam monitors the grating via specular and diffracted second-harmonic generations from the grating.

layer grating will be discussed in a separate publication. For the SHG measurements, we used the frequencydoubled beam at $0.532 \mu \mathrm{m}$ from the laser. It was incident on the sample at $71.4^{\circ}$, and covered the entire desorption area. The SHG in the specular direction was employed to monitor the average surface coverage of CO. The first-order diffraction of SHG appeared at an angle $\Delta \theta \sim 2.22^{\circ}$ from the specular direction. It was detected after spatial filtering by a photon-counting system. The fluence of the probe beam was $0.6 \sim 0.7$ $\mathrm{mJ} / \mathrm{mm}^{2}$ which caused little heating to affect the diffusion measurements. The maximum signal strength was $\sim 70$ photons per second. Both the desorption and the probe beams were incident and reflected in the horizontal plane.

The one-dimensional surface diffusion of $\mathrm{CO}$ in our case follows Fick's law:

$$
\partial \theta / \partial t=\partial(D \partial \theta / \partial t) \partial x,
$$

where $\theta$ is the surface coverage of CO. As an approximation, the diffusion constant $D$ is assumed to have the form $D=D_{0} \exp \left(-E_{\mathrm{diff}} / R T\right)$, independent of $\theta$. The solution of Eq. (1) then yields

$\theta(x)=\theta_{0}+\sum_{n=1} 2 \theta_{n} \cos (n \pi x / a) \exp \left(-n^{2} \pi^{2} D t / a^{2}\right)$.

Here, $\theta_{0}$ is the averaged coverage of $\mathrm{CO}$, and $\theta_{n}$ is the $n$th Fourier component of the monolayer grating. For $\mathrm{CO}$ adsorbed on $\mathrm{Ni}(111)$, it has been shown ${ }^{14}$ that the nonlinear susceptibility responsible for the $\mathrm{SHG}$ can be written as

$$
\chi_{s}^{(2)}=(A+C)+(B-A) \theta,
$$

where $A, B$, and $C$ refer to constants associated with the

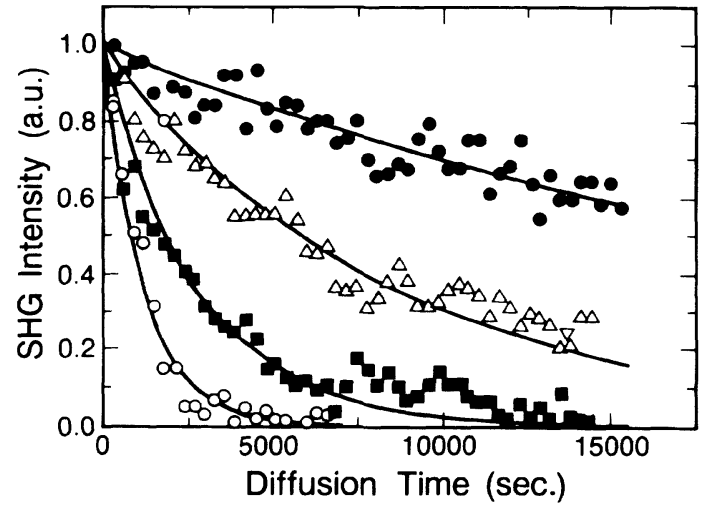

FIG. 2. Normalized first-order diffracted SHG as functions of time at various sample temperatures after laser desorption. Solid circles: $T=219 \mathrm{~K}$; open triangles: $T=247 \mathrm{~K}$; solid squares: $T=261 \mathrm{~K}$; open circles: $T=273 \mathrm{~K}$. The solid curves are least-square fits using single exponential functions with the exponent $\alpha=2 \pi^{2} D(T) / a^{2}$ as the adjustable parameter.

bare surface, the $\mathrm{CO}$-covered surface, and the bulk contribution, respectively. Since SHG is proportional to $\left|\chi_{s}^{(2)}\right|$, we have, in the specular reflection,

$$
\mathrm{SHG}_{0} \propto\left[(A+C)+(B-A) \theta_{0}\right]^{2},
$$

and, in the first-order diffraction

$$
\mathrm{SHG}_{1} \propto\left[(B-A) \theta_{1}\right]^{2} \exp \left(-2 \pi^{2} D t / a^{2}\right) .
$$

As one can see in Eq. (5), the time evolution of $\mathrm{SHG}_{1}$ following surface diffusion is independent of initial conditions, i.e., the detailed shape of the monolayer grating created.

Figure 2 shows the results of our experiment. The normalized $\mathrm{SHG}_{1}$ are plotted as functions of time at different sample temperatures. In these measurements, the readsorption was negligible as checked with the simultaneous monitoring of the specularly reflected SHG. The fluctuations on these curves were due to both shot-to-shot noise and imperfect normalization against laser beam drifts. The solid curves are the least-square fits of single exponential functions, $\exp (-\alpha t)$. Knowing $\alpha=2 \pi^{2} D / a^{2}$ from Eq. (4) and $a=10 \mu \mathrm{m}$, we can deduce the diffusion constant $D$ as a function of temperature $T$, as shown in Fig. 3. The least-square fits of the data to $D=D_{0} \exp \left(-E_{\text {diff }} / R T\right)$ gives $E_{\text {diff }}=6.9 \pm 1.0$ $\mathrm{kcal} / \mathrm{mol}$, and $\ln \left(D_{0}\right)=-11.36 \pm 2.01\left(D_{0} \sim 1.2 \times 10^{-5}\right.$ $\left.\mathrm{cm}^{2} \mathrm{~s}^{-1}\right)$.

Despite the scattering of the data, which can certainly be improved, the measured diffusion energy, $6.9 \mathrm{kcal} / \mathrm{mol}$ for $\mathrm{CO}$ on $\mathrm{Ni}(111)$, compares well with 7.0 and 7.1 $\mathrm{kcal} / \mathrm{mol}$ for $\mathrm{CO}$ on $\mathrm{Pt}(111)$ and $\mathrm{Rh}(111)$ from the hole-burning and the helium-scattering experiments. ${ }^{4}$ The preexponential factor $D_{0} \sim 1.2 \times 10^{-5} \mathrm{~cm}^{2} \mathrm{~s}^{-1}$ may seem to be somewhat small in comparison with an estimate of $4 \times 10^{-5}$ to $4 \times 10^{-4} \mathrm{~cm}^{2} \mathrm{~s}^{-1}$ from a random- 


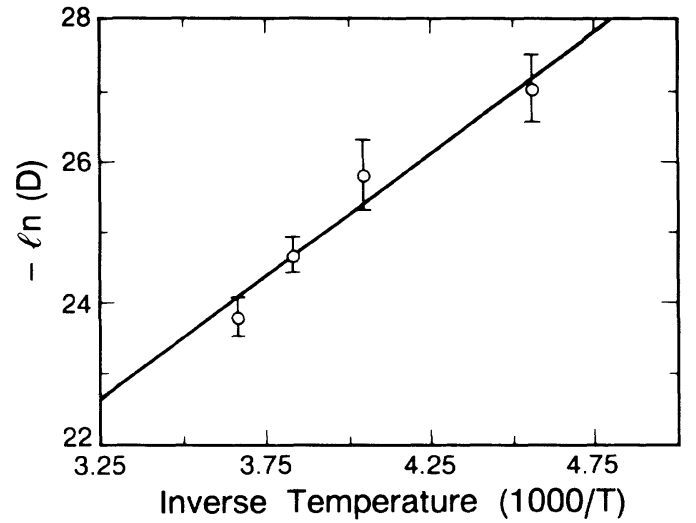

FIG. 3. The Arrhenius plot (open circles) of the diffusion constant $D(T)$ obtained from the results of Fig. 2. Leastsquare fitting to a linear function (solid line) of $1 / T$ yields the diffusion activation energy $E_{\text {diff }}=6.9 \pm 1.0 \mathrm{kcal} / \mathrm{mol}$, and the preexponential factor $D_{0}=1.2 \times 10^{-5} \mathrm{~cm}^{2} \mathrm{~s}^{-1}$ [or $\ln \left(D_{0}\right)$ $=-11.36 \pm 2.01]$.

walk model with $D_{0}=a_{0}^{2} v / 4$, taking the mean jump length $a_{0} \sim 1.25 \AA$ as the spacing between the nearest bridge sites, and the mean jumping frequency $v \sim 10^{12}$ to $10^{13} \mathrm{~s}^{-1}$ obtained from lateral vibrational frequencies of $\mathrm{Co}$ on $\mathrm{Ni}(111), 50$ to $650 \mathrm{~cm}^{-1}$. This was also found to be the case for $\mathrm{CO}$ diffusion on $\mathrm{Pt}(111)$ and $\mathrm{W}(110)$ by Seebauer, Kong, and Schmidt. ${ }^{4}$

Both the simple random-walk model and our data analysis neglected the possible coverage dependence of $D$. With $D$ being a function of $\theta$, the solution of Eq. (1) for the first Fourier component of $\theta$ is no longer a single exponential in time. If we assume $D=\alpha-\beta \theta$ to fit the data within the experimental accuracy, we find $\beta / \alpha<3$. Improvement on the signal-to-noise ratio and measurement with different initial surface coverages should yield more definite information on the coverage dependence of $\theta$.

Our technique measures macroscopic diffusion of adsorbates on a surface. It has few very attractive features. As we have seen, the analysis of the results is much more straightforward and accurate than that of the holeburning method. By varying the orientation of the grating, anisotropy of surface diffusion can be readily measured. Since the grating spacing is in the $\mu \mathrm{m}$ range and can be adjusted, the technique is capable of monitoring surface diffusion with a large dynamic range in kinetic parameters, e.g., both slow and fast diffusion processes. Finally, this method is applicable to a large variety of systems, including insulator and semiconductor surfaces.

In conclusion, we have demonstrated here a novel and promising technique for surface diffusion studies. Its simplicity, versatility, and possibility for anisotropic measurements should provide many new opportunities for research in this important area of surface science.

This work was supported by the Director, Office of Energy Research, Office of Basic Energy Sciences, Materials Sciences Division of the U.S. Department of Energy under Contract No. DE-AC03-76SF00098.

${ }^{1}$ A. G. Naumovets and Yu. S. Vedula, Surf. Sci. Rep. 4, 365 (1985).

${ }^{2}$ G. Ehlich and K. Stolt, Annu. Rev. Phys. Chem. 31, 603 (1980), and references therein.

${ }^{3}$ G. A. Somorjai, Chemistry in Two Dimensions: Surfaces (Cornell Univ. Press, Ithaca, 1981).

${ }^{4}$ E. G. Seebauer, A. C. F. Kong, and L. D. Schmidt, J. Chem. Phys. 88, 6597 (1988), and references therein.

${ }^{5}$ M. Bowker and D. A. King, Surf. Sci. 71, 583 (1978); H. Asada and M. Masuda, Surf. Sci. 99, L429 (1980); R. Morin and M. Drechsler, Surf. Sci. 111, 140 (1981).

${ }^{6}$ D. R. Bowman, Surf. Sci. 130, 348 (1983).

${ }^{7}$ G. Ehrlich, Surf. Sci. 63, 422 (1977).

${ }^{8}$ P. K. Wang, J.-P. Ansermet, S. L. Rudaz, Z. Wang, S. Shore, C. P. Slichter, and J. H. Sinfelt, Science 234, 35 (1986).

${ }^{9}$ R. Gomer, Surf. Sci. 38, 373 (1973); M. Tringides and R. Gomer, Surf. Sci. 145, 121 (1984); J. R. Chen and R. Gomer, Surf. Sci. 81, 589 (1979).

${ }^{10}$ A. P. Janssen, Surf. Sci. 52, 230 (1975).

${ }^{11}$ R. Butz and H. Wagner, Surf. Sci. 63, 448 (1977).

${ }^{12}$ R. Viswanathan, D. R. Burgess, Jr., P. C. Stair, and E. Weitz, J. Vac. Sci. Technol. 20, 605 (1982); S. M. George, A. M. DeSantolo, and R. B. Hall, Surf. Sci. 159, L425 (1985).

${ }^{13}$ H. P. Bonzel and E. E. Latta, Surf. Sci. 76, 275 (1978); P. Meakin, J. Chem. Phys. 88, 2036 (1988).

${ }^{14}$ H. W. K. Tom, C. M. Mate, X. D. Zhu, J. E. Crowell, T. F. Heinz, G. A. Somorjai, and Y. R. Shen, Phys. Rev. Lett. 52, 348 (1984); X. D. Zhu, Y. R. Shen, and R. Carr, Surf. Sci. 163, 114 (1985); H. W. K. Tom, X. D. Zhu, Y. R. Shen, and G. A. Somorjai, Surf. Sci. 167, 167 (1986); S. G. Grubb, A. M. DeSantolo, and R. B. Hall, J. Phys. Chem. 92, 1419 (1988). 